\title{
Infection by Rhodococcus equi in a patient with AIDS: histological appearance mimicking Whipple's disease and Mycobacterium avium-intracellulare infection
}

David Hamrock, Farrukh H Azmi, Edward O’Donnell, William T Gunning, Edwin R Philips, Aiman Zaher

\begin{abstract}
Rhodococcus equi pneumonia with systemic dissemination is being reported increasingly in immunocompromised patients. This is the first case report of disseminated $R$ equi infection with biopsy documented involvement of the large intestine. The patient was a 46 year old male with AIDS who was diagnosed with cavitating pneumonia involving the left lower lobe. $R$ equi was isolated in culture from the blood and lung biopsies. Subsequently, the patient developed anaemia, diarrhoea, and occult blood in the stool. Colonoscopy revealed several colonic polyps. Histological examination of the colon biopsies showed extensive submucosal histiocytic infiltration with numerous Gram positive coccobacilli and PAS positive material in the histiocytes. Electron microscopy showed variably shaped intrahistiocytic organisms which were morphologically consistent with $R$ equi in the specimen. Disseminated $R$ equi infection may involve the lower gastrointestinal tract and produce inflammatory polyps with foamy macrophages which histologically resemble those seen in Whipple's disease and Mycobacterium aviumintracellulare infection. (f Clin Pathol 1999;52:68-71)
\end{abstract}

Keywords: Rhodococcus equi; AIDS; colonic disease

Rhodococcus equi, previously known as $\mathrm{Co}$ rynebacterium equi, is an aerobic, Gram positive, mildly acid-fast coccobacillus. ${ }^{1}$ Originally isolated from foals with pneumonia in 1923, this organism is well known today by veterinarians to be a cause of pulmonary abscesses and lymphadenitis in several animal species. ${ }^{12}$ Since the first reported case of Rhodococcus equi in humans in 1967, there have been several additional reports of pneumonia caused by this organism. ${ }^{134}$ However, only a small minority of these case reports demonstrating pneumonia also showed pathology in organs outside of the respiratory system. ${ }^{3}{ }^{5-8}$ Furthermore, only one case of probable gastrointestinal involvement by $R$ equi has been reported previously. ${ }^{8}$ As far as we know, this is the first case which histologically showed the presence of $R$ equi within histiocytes densely infiltrating the submucosa of the large intestine.
Case report

A 46 year old HIV positive white male was admitted to the Medical College of Ohio hospital in late October 1996, complaining of fever, night sweats, dry cough, and weight loss. Previously, the patient underwent a bronchoscopy in May 1996 in response to a mass in the left lower lobe discovered on routine chest $x$ ray. A culture at the time of bronchoscopy grew $R$ equi, which was sensitive to penicillin, erythromycin, trimethoprim-sulphamethoxazole (TMP-SMX), rifampicin, and vancomycin. It was resistant to clindamycin. His clinical condition improved moderately but not fully, after taking TMP-SMX, rifampicin, and clarithromycin daily for several months.

Biopsies from a subsequent bronchoscopy in September 1996 showed a severe active, chronic pneumonitis with Gram positive coccobacilli within foamy histiocytes. $R$ equi grew from cultures of these transbronchial biopsies as well as from bronchial washings. Three blood cultures drawn at the same time also grew $R$ equi. All of these bacterial isolates from both the lung and blood cultures were sensitive to the same antibiotics as in May 1996, except that all isolates were now uniformly resistant to penicillin.

Because the lung lesion had not completely resolved and the patient was still having cough and fever despite taking the antibiotics mentioned, it was agreed to resect the affected lobe. A presurgical evaluation showed mild anaemia and occult blood present in the stool. The patient also complained of chronic diarrhoea which began in September 1996 at the time when blood cultures were positive for $R$ equi. Colonoscopy in early November 1996 showed several "colonic polyps" approximately $10 \mathrm{~mm}$ in size which were excised. Histological examination showed inflammatory polyps with Gram positive coccobacilli. Two days later, the patient underwent an operation to resect the pathological left lower lobe. A culture of this tissue grew $R$ equi which again was sensitive to TMP-SMX, erythromycin, rifampicin, and vancomycin. It was resistant to penicillin and clindamycin. After an uncomplicated postoperative course, the patient's pulmonary function improved markedly.

While in the hospital, the patient slowly began losing his short term memory. Magnetic resonance imaging of the brain showed several enhancing lesions in both hemispheres. In addition to his current three-antibiotic regi-
Accepted for publication 25 August 1998 

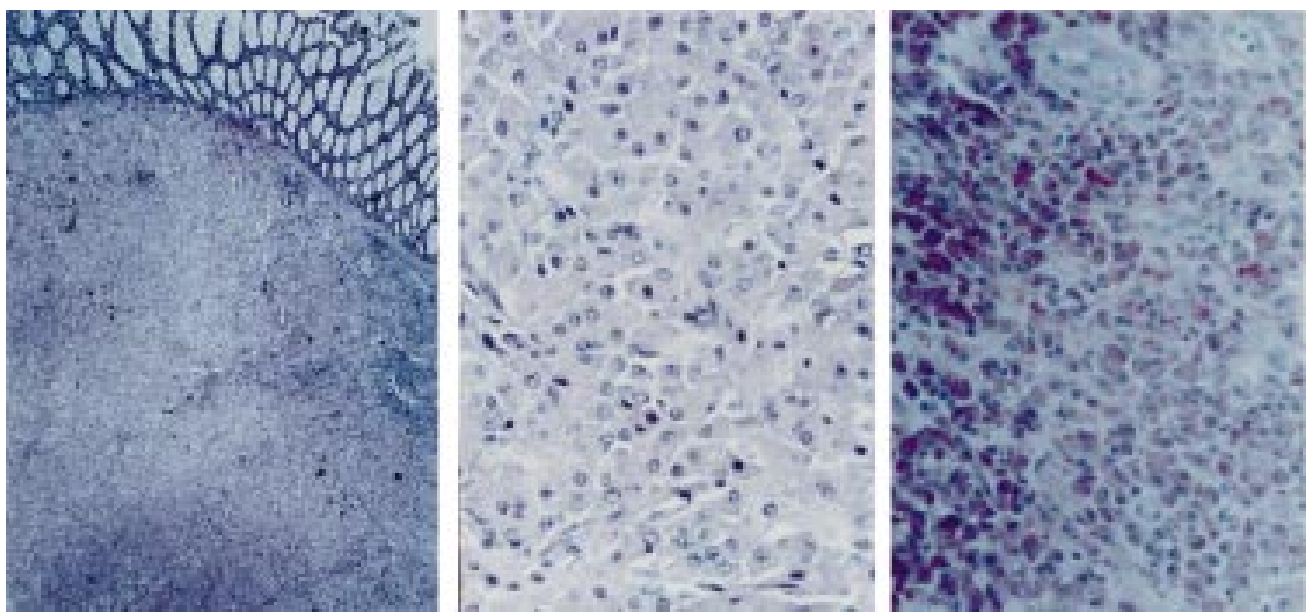

Figure 1 Endoscopic colon biopsy showing extensive infiltration of foamy histiocytes with abundant eosinophilic PAS positive cytoplasm, Left, HEE stain low power; middle, $H \mathcal{E} E$ stain high power; right, PAS stain.

men, pyrimethamine was added to cover for possible infection by Toxoplasma gondii. In December 1996, computed tomography (CT) of the brain was performed because of the onset of seizure activity. This scan showed several additional ring enhancing lesions. Vancomycin and clindamycin were added for additional $R$ equi and Toxoplasma gondii coverage, respectively. At a subsequent hospital admission in February 1997, multiple biopsies were obtained from several of the brain lesions to determine their cause. These samples showed an inflammatory microangiopathy on routine haematoxylin and eosin stains. A Gram stain revealed scant Gram positive rod shaped microorganisms consistent with $R$ equi. No Toxoplasma gondii organisms were seen. A repeat CT scan of the brain showed that the lesions were resolving after approximately one month of vancomycin. Subsequently, all antibi- otics were discontinued except for vancomycin and rifampicin. After two months of this treatment, his neurological symptoms had resolved and another CT scan of the brain showed that nearly all the brain lesions had cleared. There was also no recurrence of pneumonia.

\section{Pathological findings}

All three biopsies taken from the caecum, ascending colon, and transverse colon showed massive infiltration of histiocytes within the submucosa (fig 1). These histiocytes had abundant eosinophilic cytoplasm which stained strongly with periodic acid Schiff (PAS) stain. A Gram stain revealed many intracytoplasmic Gram positive coccobacilli. A silver stain for fungal organisms was negative, and an acid-fast stain was unremarkable in that only a few organisms stained faintly positive. Electron microscopy studies revealed oval shaped bacte-

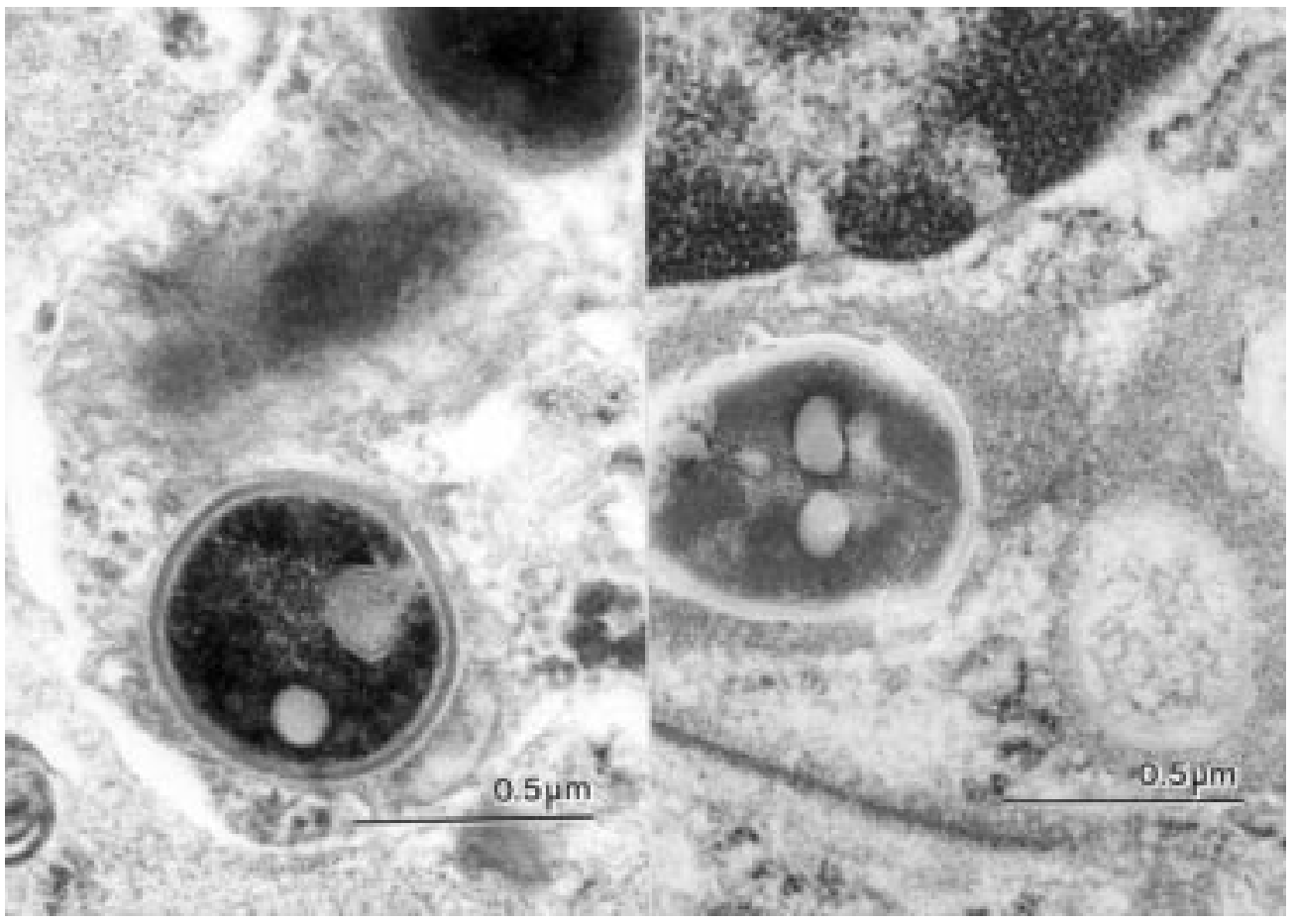

Figure 2 Transmission electron microscopy of colon biopsy (right) and lung biopsy (left), showing intracytoplasmic coccobacilli with thick cell walls and lipid vacuoles ( $\times 59325)$. 

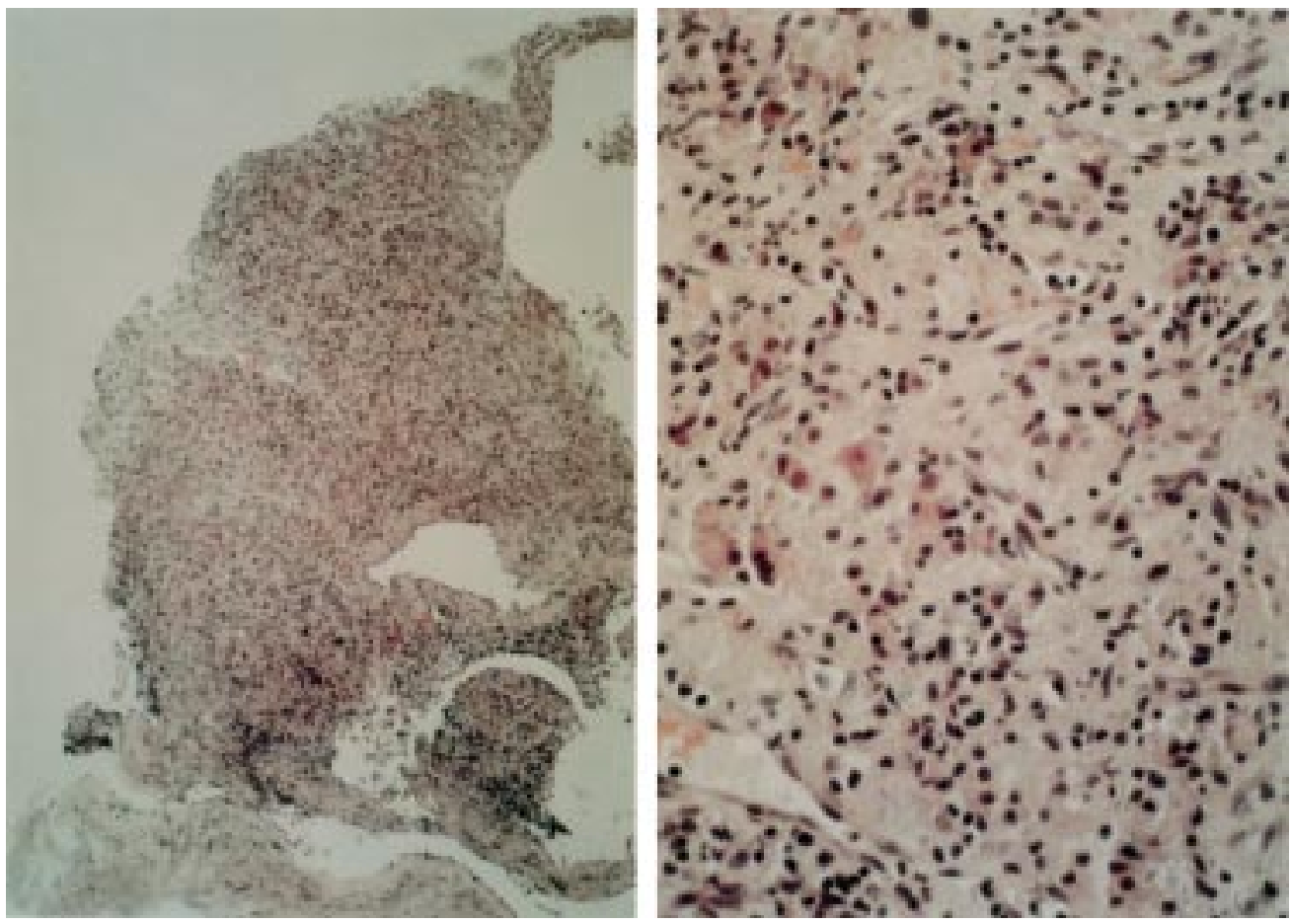

Figure 3 Transbronchial lung biopsy showing obliteration of lung parenchyma by extensive infiltration of foamy histiocytes with abundant eosinophilic cytoplasm. Left, HEE stain low power; right, HEE stain high power.

ria with a thick cell wall and multiple lipid vacuoles, very similar to the morphology of mycobacteria (fig 2). There were no aggregates of membranous material to suggest that the organisms were Whipple's bacilli.

The left lower lobe of the lung measured $13.0 \times 11.0 \times 6.0 \mathrm{~cm}$. and weighed $630 \mathrm{~g}$. The pleural surface had many fine adhesions and a $5.0 \times 4.5 \mathrm{~cm}$. area of subpleural haemorrhage was also present. The entire lobe was consolidated by a soft, creamy, yellowish-tan pasty material. Architecturally, the lobe resembled a liver with macronodular cirrhosis. Microscopically, there was obliteration of the normal lung parenchyma with dense infiltration of histiocytes. The outline of alveoli was observed only occasionally, and when they were seen, they were nearly filled with histiocytes. Similar to those in the intestinal wall, these histiocytes had abundant, eosinophilic, globular cytoplasm (fig 3). A Gram stain showed the presence of Gram positive coccobacilli within the cytoplasm of the histiocytes. The organisms did not stain definitively with an acid-fast stain, and a silver stain for fungal organisms was negative. These histiocytes were likewise strongly PAS positive. Electron microscopy of a portion of the lung tissue showed many intracytoplasmic coccobacilli with thick cell walls and intracytoplasmic lipid vacuoles (fig 2). No Michaelis-Gutmann bodies were identified to suggest malakoplakia. As mentioned cultures from this tissue grew $R$ equi.

\section{Discussion}

Human infection with $R$ equi is now increasingly being reported in immunocompromised patients as a result of HIV infection, haematological malignancies, and immunosuppression for transplantation. ${ }^{36}$ Rare cases have also been reported in patients with functioning immune systems. ${ }^{3} R$ equi is usually transmitted by inhalation and commonly produces a cavitating pneumonia. ${ }^{1}$ So far as we know, this is the first report describing a case of disseminated $R$ equi infection with histologically documented involvement of the gastrointestinal tract.

When there is intense infiltration of foamy eosinophilic histiocytes within the wall of the intestine, Whipple's disease and infection by Mycobacterium avium-intracellulare are two main infections which should be considered in the differential diagnosis. ${ }^{9}{ }^{10}$ With this case, we show that the inflammatory response elicited by $R$ equi in the intestine produces a near identical histological picture to Mycobacterium avium-intracellulare infection and Whipple's disease. Culture of the colon biopsies were not ordered. However, the morphological appearance of the organisms by light and electron microscopy was identical to those seen in lung biopsies which grew $R$ equi. Furthermore, the documentation of systemic spread was confirmed by positive blood cultures. Indeed, an acid-fast stain, which would be expected to be strongly positive in Mycobacterium aviumintracellulare infection, was weakly positive in focal areas only, consistent with $R$ equi. Although the histiocytes were strongly PAS positive, electron microscopy failed to reveal the characteristic Tropheryma whippelii bacilli seen in Whipple's disease. In addition, Whipple's disease involving the gastrointestinal tract more often affects the small rather than the large intestine. ${ }^{11}$ Also, Gram staining showed numerous intracytoplasmic Gram positive coccobacilli whereas $T$ whippelii is Gram negative. ${ }^{9}$

In conclusion, $R$ equi infection should be included in the differential diagnosis along with Whipple's disease and Mycobacterium avium- 
intracellulare whenever foamy eosinophilic histiocytes are seen in colon biopsies from an immunocompromised patient.

1 Prescott JF. Rhodococcus equi: an animal and human pathogen. Clin Microbiol Rev 1991;4:20-34.

2 Prescott JF. Epidemiology of Rhodococcus equi infection in horses. Vet Microbiol 1987;14:211-14.

3 Scott M, Graham BS, Verrall R, et al. Rhodococcus equi-an increasingly recognized opportunistic pathogen. Am F Clin Pathol 1995;103:649-55.

4 Frame BC, Pektus AF. Rhodococcus equi pneumonia: case report and literature review. Ann Pharmacother 1993;27: report and $1340-2$.

5 Antinori S, Esposito R, Cernuschi M, et al. Disseminated Rhodococcus-equi-infection initially presenting as foot mycetoma in an HIV-positive patient. AIDS 1992;6:740-2.
6 Harvey RL, Sunstrum JC. Rhodococcus equi infections in patients with and without human immunodeficiency virus infection. Rev Infect Dis 1991;13:137-45.

7 Franklin DB, Jackson YJ, Hawkins SS. Corynebacterium equi peritonitis in a patient receiving peritoneal dialysis. South Med f 1989;82:1046-7.

8 Fierer J. Non-pulmonary Rhodococcus equi infections in patients with acquired immune deficiency syndrome (AIDS). F Clin Pathol 1987;40:556-8.

9 von Herbay A, Maiwald M, Ditton HF, et al. Histology of intestinal Whipple's disease revisited. Virchows Arch 1996; 429:335-43.

10 Poorman JC, Katon RM. Small bowel involvement by Mycobacterium avium complex in a patient with AIDS: endoscopic, histologic, and radiographic similarities to Whipple's disease. Gastrointest Endosc 1994;40:753-9.

11 Marth T, Strober W. Whipple's disease. Semin Gastrointest Dis 1996;7:41-8.

\section{CALL FOR COVER ILLUSTRATIONS}

Readers with material that might be suitable for the front cover of the Journal are invited to submit prints or transparencies to the editor. Description and acknowledgement is published inside the front cover. This could be a good way of getting your work seen! 